\title{
A new luminescence detection and stimulation head for the Risø TL/OSL reader
}

Lapp, Torben; Kook, Myung Ho; Murray, Andrew Sean; Thomsen, Kristina Jørkov; Buylaert, Jan-Pieter; Jain, Mayank

Published in:

Radiation Measurements

Link to article, DOI:

10.1016/j.radmeas.2015.02.001

Publication date:

2015

Document Version

Peer reviewed version

Link back to DTU Orbit

Citation (APA):

Lapp, T., Kook, M. H., Murray, A. S., Thomsen, K. J., Buylaert, J-P., \& Jain, M. (2015). A new luminescence detection and stimulation head for the Risø TL/OSL reader. Radiation Measurements, 81, 178-184.

https://doi.org/10.1016/j.radmeas.2015.02.001

\section{General rights}

Copyright and moral rights for the publications made accessible in the public portal are retained by the authors and/or other copyright owners and it is a condition of accessing publications that users recognise and abide by the legal requirements associated with these rights.

- Users may download and print one copy of any publication from the public portal for the purpose of private study or research.

- You may not further distribute the material or use it for any profit-making activity or commercial gain

- You may freely distribute the URL identifying the publication in the public portal

If you believe that this document breaches copyright please contact us providing details, and we will remove access to the work immediately and investigate your claim. 


\title{
The new luminescence detection and stimulation head for the Risø TL/OSL reader
}

T. Lapp ${ }^{1 *}$, M.H. Kook ${ }^{1}$, A.S. Murray², K.J. Thomsen ${ }^{1}$, J.-P. Buylaert ${ }^{1,2}$, M. Jain ${ }^{1}$

1. Center for Nuclear Technologies, Technical University of Denmark, DTU Ris $\varnothing$ Campus, Denmark

2. Nordic Laboratory for Luminescence Dating, Department of Geoscience, Aarhus University, Ris $\varnothing$ Campus, DK-4000 Roskilde, Denmark

*Corresponding author: T.Lapp, phone: +45 2132 6231, email: tlap@dtu.dk

\begin{abstract}
A new automated Detection And Stimulation Head (DASH) has been developed for the Ris $\emptyset$ TL/OSL luminescence reader to provide easy access to new technologies, new signals and new measurement methods. The automated DASH includes a filter changer and a detector changer that makes it possible to change stimulation filters ( $4 \times 4$ filter combinations possible) and detectors ( 3 detectors possible) as part of a measurements sequence. The new automated DASH with dedicated driver electronics, can be retrospectively fitted to existing Ris $\varnothing$ TL/OSL readers not excluding any of the previously developed attachments.
\end{abstract}

\section{Introduction}

The basic platform of the Ris $\varnothing$ TL/OSL reader includes heating, optical stimulation detection and irradiation (e.g. Bøtter-Jensen et al. 2010) and is widely used in many research laboratories across the world. The platform also serves as a basis for a variety of attachments, including a single-grain measurement system, optical camera, x-ray source, radio-luminescence facility and others (Bøtter-Jensen et al. 2003, Lapp et al. 2009, Lapp et al. 2012).

Here we describe a new DASH containing modules of blue $(470 \mathrm{~nm})$, green $(525 \mathrm{~nm})$ and infrared $(870 \mathrm{~nm})$ LEDs, two automated filter changers and a detector changer; this new facility is intended to significantly increase the flexibility in both routine and research measurements. The main reasons for developing this new facility are:

1. To allow for automated changing of filters within a sequence, which is particularly useful for feldspar dating or working with mixed minerals. 
2. To provide automated change of detectors. This is intended to facilitate comparative studies of different detection ranges, and allows the inclusion of a high sensitivity camera (EMCCD) to explore the potential of luminescence imaging.

We also regard it as important that the new head can be retrospectively fitted to previous versions of the reader platform. All readers with turntable diameter of $\mathrm{xx} \mathrm{mm}$ (introduced 19xx) may potentially be upgraded.

The new DASH consists of:

1. Driver and control board (plug-in board for the Reader Controller)

2. Base unit containing stimulation LEDs, feed-back diodes, collimating optics, memory, and controller for the EMCCD camera focusing optics.

3. Two stacked filter changers (4 filters in each)

4. Detector changer (3 positions)

Each unit has an embedded controller that can store setup and status information and all these controllers are mastered by the Ris $\varnothing$ TL/OSL Controller via the driver board. A block diagram and the DASH mounted on a reader are shown in Figure 1

Figure 1

\section{Filter- and detector changers}

The automated DASH has two stacked filter changers, making it possible to combine filters (see also Richter et al., 2013). Each filter changer has 4 positions, making 16 filter combinations possible. The filter carrier is designed to accept filters of up to $25 \mathrm{~mm}$ diameter and the filter (or stack of filters) may be up to $8 \mathrm{~mm}$ thick. This diameter is a standard size making it easy to use commercially available filters for special purposes. When the filters are mounted in the filter changers they are identified in the Sequence Editor setup by their name and unique ID number, so that when sequences (the sequence of operations) are stored, the filter IDs selected for the particular operation are also stored. This allows the transfer of sequences from one reader to another even if filters are not mounted in the same positions. User-defined filters may also be specified, and again if the same user defined ID's are used in several readers, sequences are directly interchangeable between readers no matter if the filters are mounted in the same positions.

The detector changer has three positions for different detectors. All detectors use a common collimating lens system with an aperture of $25 \mathrm{~mm}$ diameter and a focal length of $25 \mathrm{~mm}$ (see Figure 2). If the detector changer has an EMCCD camera mounted, this common lens system is a fused silica based lens system intended to provide a good compromise between imaging characteristics and optical transmission (see 
Kook et al., these proceedings). If imaging is not required, a simpler fused-silica based optics optimised for maximum transmission is used.

The standard detector is an Electron Tube 1" PMT module based on an EMD-9107 PMT (160-630 nm) with quartz window, selected for low background $\left(<100 \mathrm{~s}^{-1}\right)$. Because the collimating optics collects the light from a large solid angle, the sensitivity of this detector system is somewhat greater than that of the classic DASH (about 30\%)

An optional detection unit is a red sensitive PMT unit based on a Hamamatsu H7421-50 (cooled to $0^{\circ} \mathrm{C}, 380$ $890 \mathrm{~nm}$ ) PMT module with optics that interfaces to the $5 \mathrm{~mm}$ diameter active area of the PMT.

Another optional detection unit is a high sensitivity EMCCD camera (described in a separate paragraph below). This unit includes an automated adjustable focusing optics.

Given the degree of flexibility and automation it is important to ensure that combinations of filters and light sources that may damage a detector cannot be chosen. The allowed combinations of light sources, filters and detectors are specified in the Sequence Editor.

To illustrate one of the advantages of the flexibility provided by this unit, we have measured 3 quartz samples (115406-28 and -30). The first sample is colluvium from Colorado, and is well-bleached. The other two are colluvium from Germany, and are thought to be poorly bleached. All samples passed the standard IR depletion ratio test (Duller at al., 2003). We have measured the quartz extracted from these samples using the $I_{50}$ (in blue) and OSL (in UV) signals in a so-called double SAR quartz protocol (Banerjee et al., 2001). Conventionally, the IR signal in a double SAR protocol is measured in the UV, but here we are able to measure with a blue filter in the same sequence. Because IR signals are much less sensitive to daylight than quartz, the ratio of the $\mathbb{I}_{50}$ dose to the quartz dose gives direct information about how well these quartz grains were bleached (Murray et al., 2012). In this case, we are measuring small feldspar inclusions in sandsized quartz grains and so it can be safely assumed that there will be no significant contribution from internal ${ }^{40} \mathrm{~K}$. Thus we expect for well-bleached material that the feldspar to quartz dose ratio should be $\sim 0.7$ (assuming a typical $3 \% /$ decade anomalous fading); if the ratio is significantly $>1$, we would deduce that the grains might not be well-bleached. For these samples, the IR Fo $_{5}$ OSL dose ratios are 0.6, 2.6 and 2.6, respectively; the separation between well-bleached and poorly-bleached sample is very clear.

\section{Stimulation unit}

LEDs are chosen for optical stimulation mainly because of their long lifetime and stability. In the classic DASH (e.g. Bøtter-Jensen et al., 2010) optical stimulation was achieved by using 4 blue LED clusters and 3 IR LED clusters; each cluster consisting of 7 LEDs. In the automated DASH we have used powerful LEDs with parabolic reflectors (Epitex SMBB470-1100-TINA-RS, SMBB525-1100-TINA-RS, SMBB870-1100-TINA-RS). 
The stimulation layer of the DASH contains 7 holes for LEDs with reflectors and one hole for single grain laser beam entry (Duller et al, 1999) or another external stimulation light source. This extra light source may be e.g. a violet $405 \mathrm{~nm}$ laser (Jain et al., 2009) or a user-supplied stimulation source. The 8 holes are evenly distributed around the periphery of a circle with the sample location at its centre. The beams from the individual LEDs aim towards the centre of the sample at an incident angle of $45^{\circ}$ (see Figure 2 ), and the standard configuration contains: 2 blue $\left(470 \mathrm{~nm}, 100 \mathrm{~mW} / \mathrm{cm}^{2}\right), 2$ green $\left(530 \mathrm{~nm}, 50 \mathrm{~mW} / \mathrm{cm}^{2}\right)$ and $3 \mathrm{IR}$ $\left(870 \mathrm{~nm}, 175 \mathrm{~mW} / \mathrm{cm}^{2}\right)$. The head may be configured with other combinations, e.g. 4 Blue $+3 \mathrm{IR}$, or 4 Green +3 IR LEDS

Figure 2

The LEDs are mounted in close thermal contact with the DASH aluminium framework to provide good thermal contact with the entire reader and so ensure long lifetime and stability for the LEDs. Three of the LED positions (normally one blue, one green and one IR) have a feed-back photodiode mounted for regulation of the power during stimulation.

We have made a comparison of quartz and feldspar OSL decay curves measured on the existing and the new head using blue and IR stimulation. The decay curves shown in Figure 3 are acquired from the same aliquot and with identical dose and pre-treatment. The new DASH delivers a somewhat higher effective stimulation power both in blue (approx. 10\% increase) and IR (approx. 30\% increase), in a new DASH that is configured for blue, green and IR stimulation.

Figure 3

The uniformity of the stimulation light field has been measured by analysis of digital images of the three types of stimulation. The three types of LEDs were turned on to illuminate a white reflecting screen placed in the sample plane. An $11 \mathrm{~mm}$ diameter circle was drawn centred on the sample location for geometrical calibration. The images were converted to RGB bitmaps; for the $470 \mathrm{~nm}$ blue stimulation the $B$ pixel value was used, for the green $525 \mathrm{~nm}$ stimulation the $\mathrm{G}$ pixel value, and for the IR stimulation the R pixel value. For all experiments we ensured that the light intensities were far from the saturation level of the image sensor, and the linearity of the pixel values was tested for the blue and green stimulation. The IR stimulation image is acquired with a simpler camera without IR filter; this does not allow manual exposure setting and so linearity could not been checked for IR acquisition. Pixel data from the circular part of the picture, and contour plots of the intensity of the 3 stimulation wavelengths within a $10 \mathrm{~mm}$ diameter area are shown in Figure 4a-c. Profiles along the direction with the lowest variation (major axis) and along the perpendicular direction with the highest variation (minor axis) are shown in Figure $4 d$ 
We have measured the standard deviation of the illumination intensity of 3 different sample size diameters: $5 \mathrm{~mm}, 8 \mathrm{~mm}$ and $10 \mathrm{~mm}$. These results are shown in Table 1 together with the standard deviations for the same sample diameters of the classic DASH obtained using a similar measurement procedure.

In case of the blue and green LEDs, illumination is more uniform along the major axis of the illumination ellipse compared to the minor axis direction perpendicular to it (Figs $4 a, b, d)$. The maximum difference between the central maximum and the periphery minimum along the two main axis's are also shown in table 1.

Table 1

The results for the dispersion in illumination intensity show a significant improvement compared with the classic DASH, although the absolute measure of the deviation of the IR illumination needs to be confirmed after testing the linearity of the acquisition. Local deviations in illumination intensity are observed in the images in Figure $4 a-c$, however these are over too short distances to be likely to arise from the light sources and may be due to local variation in reflectivity of the projection screen.

\section{Driver board and control boards}

The OSL and pulsed OSL (POSL) driver boards for the existing TL/OSL reader head (plug-in boards in the Ris $\varnothing$ TL/OSL Controller) have been replaced by a single OSL and POSL driver and control board. The integration of the two OSL and POSL functions makes it possible to mix standard OSL and POSL measurements in the same sequence. In addition the restrictions on the possible combinations of on-time, off-time and gating intervals that arose in the old system have been removed, and parameters may now be freely specified with a resolution of $0.1 \mu \mathrm{s}$ in the range from $1 \mu \mathrm{s}$ to $10 \mathrm{~s}$.

The board also contains a PID regulation of the power during stimulation. For short pulses the regulation is based on an integration of a photodiode feedback signal over a number of pulses. For long pulses and continuous stimulation the regulation is based on the integration of the feed-back signal over successive parts of the on-time.

The driver board also contains a Local Interconnect Network (LIN)-bus master that communicates with 4 control boards in the DASH itself: 1) base unit storing calibration of the head and controlling the focusing unit of the EMCCD camera, 2) and 3) filter changer units that change the filter positions and store the current position, 4) detector changer unit that changes the detector position and stores the current position.

When the DASH is first assembled, an initial feed-back calibration is measured and stored in the head. This feed-back calibration stores, for each stimulation type, the feed-back signal measured at $100 \%$ power level, 
and the absolute maximum permitted voltage and current that can be used to drive the LEDs. A power calibration can be initiated subsequently from the Control program to establish a look-up table for the initial voltage for different power settings. This ensures that the power is at the correct setting immediately the LEDs are switched on, rather than allowing some period for stabilisation depending on the effective time constant of the feedback circuit. This power calibration procedure is fully automated, and may be repeated regularly if desired.

\section{High sensitivity camera (EMCCD)}

Sensitive cameras for imaging luminescence signals have been tested in the past (Duller et al, 1997, Greilich et al., 2002, Greilich and Wagner, 2006). Now with the availability of the EMCCD (Electronically Magnified Charge Coupled Device) camera there has been a renewed interest in this option (Clarc-Balzan and Schwenninger 2012, Richter et al., 2013, Mundupuzhaka et al., 2014). The new DASH presented here includes a detector position for a back-thinned, Peltier cooled $\left(-80^{\circ} \mathrm{C}\right)$ Photometrics Evolve $512 \mathrm{EMCCD}$ camera (Kook et al., these proceedings). The EMCCD camera has been used for single grain measurements, and a comparison with the standard single grain system with laser scanning has been undertaken (Thomsen et al., these proceedings). The EMCCD camera also provides additional measurement possibilities that are not available with the standard single grain attachment: 1 ) it can acquire spatially resolved TL luminescence measurements; 2 ) it can examine the luminescence from arbitrary Region Of Interest (ROI) in TL/OSL images from large specimens, e.g. rock slices. Duller et al. (these proceedings) has tested this potential application by measuring the TL signals from biogenic carbonate samples, and using these to derive the formation time of snail opercula.

The main challenge in using an image to derive dose distributions is the automatic reduction of the data so that the luminescence from a particular ROI is reproducibly integrated from one measurement to the next (as is for instance required for the generation of a SAR dose response curve). In the case of an OSL signal, this process begins with the collection of a sequence of image frames during the decay of the luminescence. After the collection of each signal the sample is illuminated for a short period and an optical image is obtained. This image is used for defining the ROIs and for measuring any displacement of the sample from one acquisition to the next. The measurement of displacement is based on 3 optically contrasting marks. In the case of single grain measurements, the calculation is based on the 3 marker holes drilled through the sample disc. The sample disc also contains holes for the individual grains that ensure that the individual grains do not move relative to the marker holes. Using the manually or automatically selected ROIs and the position corrected individual frames, the signals are integrated spatially, to provide the time dependent change in luminescence for a particular ROI. These data are then stored as a standard single grain BIN file, where the grain number now identifies the ROI number. This BIN file may then be 
analysed in the same manner as any standard single grain data file by use of e.g. the program Analyst (freely downloadable from www.osl.risoe.dk).

An example of TL glow curves from two different regions (ROIs) on a granite rock slice is shown in Figure 5. The rock slice has been irradiated with about $35 \mathrm{~Gy}$ and TL luminescence images have been acquired in 3 different wavelength regions: blue, green-yellow, and red. Glow curves have been derived from the luminescence images for 2 different ROIs, and especially for the green-yellow detection range a significant difference between the two regions may be observed.

The full potential of such spatially resolved TL luminescence measurements has only just begun to be investigated. As examples of the applications may be mentioned: Application to biogenic carbonate (Duller et al., these proceedings), Kook et al. (these proceedings) show the correlation between the $110^{\circ} \mathrm{C} T \mathrm{~L}$ peak obtained during preheat, with the OSL signal obtained after preheat; this correlation has considerable potential in single grain sensitivity correction (Spooner, N.A., 1994).

Figure 5

\section{Conclusion}

The detection and stimulation head (DASH) of the Ris $\varnothing$ TL/OSL reader has been upgraded to allow automated changing of both filters and detectors during an acquisition sequence. This new configuration is slightly more sensitive than the earlier system and the stimulation intensity is slightly greater. The flexibility offered by the new DASH allows, for example, feldspar signals emitted at blue wavelengths to be collected in the same sequence as UV signals from quartz. This has obvious application for quartz contaminated with feldspar, amongst others. In addition to the two photomultiplier tube positions, a new EMCCD camera imaging system has been mounted on the detector changer, to allow TL and OSL imaging. This camera may be used for either single grain or ROI-based TL and OSL data acquisition. Routine data collection and SAR analysis of such single grain or 'ROI' data extracted from these images can be carried out in the usual manner using Analyst. Table 2 gives an overview of the stimulation sources, standard filters and detectors available at present.

Table 2

\section{Acknowledgements}

The development of the automated DASH has been a joint effort of the engineering group at DTU Nutech: Henrik Esmann Christiansen, Søren Vig Dalsgaard, Jørgen Hesselbjerg Jakobsen, Myungho Kook, Lars Pirtzel, Per Günther Sørensen. 


\section{References}

Banerjee, D., Murray, A.S., Bøtter-Jensen, L., Lang, A., 2001. Equivalent dose estimation using a single aliquot of polymineral fine grains. Radiation Measurements 33, 73-94.

Bøtter-Jensen, L., Andersen, C.E., Duller, G.A.T., Murray, A. S., 2003. Developments in radiation, stimulation and observation facilities in luminescence measurements, Radiation Measurements, Volume 37, Issues 4-5, August-October 2003, Pages 535-541.

Bøtter-Jensen, L., Thomsen, K.J., Jain, M., 2010. Review of optically stimulated luminescence (OSL) instrumental developments for retrospective dosimetry. Radiation Measurements 45, 253-257.

Clark-Balzan, L., Schwenninger, J-L., 2012. First steps toward spatially resolved OSL dating with electron multiplying charge-coupled devices (EMCCDs): System design and image analysis. Radiation Measurements $47,797-802$

Duller, G.A.T., Bøtter-Jensen, L., Markey, B.G., 1997. A luminescence imaging system based on a CCD camera Radiation Measurements 27, 91-99.

Duller, G.A.T., Bøtter-Jensen, L., Murray, A. S., 2000. Optical dating of single sand-sized grains of quartz: Sources of variability. Radiation Measurements 32, 453-457.

Duller, G.A.T., 2003. Distinguishing quartz and feldspar in single grain luminescence measurements. Radiation Measurements 37, 161-165.

Duller, G.A.T., Kook, M.H., Stirling, R. J., Roberts, H.M., Murray, A.S., Spatially resolved thermoluminescence from snail opercula using an EM-CCD, These proceedings

Greilich, S., Glasmacher, U.A., Wagner, G.A., 2002. Spatially resolved detection of luminescence: a unique tool for archaeochronometry. Naturwissenschaften 89, 371-375.

Greilich, S., Wagner, G., 2006. Development of a spatially resolved dating technique using HR-OSL. Radiat. Meas. 41, 738-743.

M. Kook, T. Lapp, A.S. Murray, K.J. Thomsen, M. Jain, A luminescence imaging system for the routine measurement of single-grain OSL dose distributions, These proceedings

Jain, M., 2009. Extending the dose range: probing deep traps in quartz with $3.06 \mathrm{eV}$ photons. Radiat. Meas. 44, 445-452.

Mundupuzhakal, J., Adhyaru, P., Chauhan, N., Vaghela, H., Shah, M., Chakrabarty, B., Acharya, Y., 2014. FPGA based TL OSL system with EMCCD for luminescence studies. 2014 JINST 9 P04001.

Murray, A.S, Thomsen, K.J., Masuda, N., Buylaert, J.P., Jain, M., 2012. Identifying well-bleached quartz using the different bleaching rates of quartz and feldspar luminescence signals. Radiation Measurements 47, 688696.

Lapp, T., Jain, M., Thomsen, K.J., Murray, A.S., Buylaert, J.P., 2012. New luminescence measurement facilities in retrospective dosimetry. Radiation Measurements 47, 803-808.

Lapp, T., Jain, M., Ankjærgaard, C., Pirtzel, L., 2009. Development of pulsed stimulation and Photon Timer attachments to the Ris $\varnothing$ TL/OSL reader. Radiation Measurements 44, 571-575. 
Richter, D., Richter, A., Dornich, K., 2013. Lexsyg - a new system for luminescence research.

Geochronometria 40, 220-228

Spooner, N.A., 1994. On the optical dating signal from quartz. Radiat. Meas. 23, 593-600., Bailey, R., 1997.

Optical detrapping of charge from the $110^{\circ}$ quartz TL region. Ancient TL 15, 7-10.

Thomsen, K.J., Kook, M.H., Murray, A.S., Jain, M., Lapp, ${ }^{1}$, Single-grain results from an EMCCD-based imaging system, These proceedings 


\section{Figure and table captions}

Figure 1 a) A picture of the automated DASH mounted onto the TL/OSL Reader. b) A block diagram how the DASH units are controlled by the DASH driver board mounted in the TL/OSL Controller.

Figure 2 Cross section of the DASH base unit. 1) Stimulation LED with reflector, 2) LED cooler 3) stimulation filter, 4) feed-back photodiode, 5) sample heater, 6) collimating optics.

Figure 3 Comparison of classic and automated DASH efficient stimulation power by decay curves obtained by similar conditions with the two heads. a) Blue stimulation, b) IR stimulation.

Figure 4 Contour plots of illumination intensity of the 3 different stimulation wavelengths.

Figure 5 Example of glow curves for 2 different ROls of a granite rock slice (Dose: 35 Gy) in 3 different detection ranges. a) Picture of the Rock slice with indication of the ROIs. b) Glow curves. c-d) Luminescence images in the green-yellow range at the temperatures $t_{1}$ and $t_{2}$ as indicated in $b$ ).

Table 1 Variation in 3 different sample diameter regions and compared with the variations for the classic stimulation head. Major axis angles are: Blue: $-45^{\circ}$, Green: $+45^{\circ}$, IR: $0^{\circ}$. Minor axis is perpendicular to the major axis. Standard deviations are relative to the mean, (Max-Min) are relative to Max.

Table 2 Overview of stimulation sources, filters and detectors available 
Table 1

\begin{tabular}{|l|l|l|l|l|l|}
\hline $\begin{array}{l}\text { Sample } \\
\text { dia- } \\
\text { meter }\end{array}$ & $\begin{array}{l}\text { Stimulation } \\
\text { wavelength }\end{array}$ & $\begin{array}{l}\text { Relative } \\
\text { std. } \\
\text { dev. }\end{array}$ & \multicolumn{2}{l|}{$\begin{array}{l}\text { Relative } \\
\text { (Max-Min) } \\
{[\%]}\end{array}$} & $\begin{array}{l}\text { Relative } \\
\text { std. dev. } \\
\text { (classic } \\
\text { DASH) } \\
{[\%]}\end{array}$ \\
\cline { 3 - 6 } & {$[\mathrm{nm}]$} & $\begin{array}{l}\text { Major } \\
\text { axis }\end{array}$ & $\begin{array}{l}\text { Minor } \\
\text { axis }\end{array}$ & \\
\hline \multirow{5}{*}{5} & 470 & 2.0 & 4 & 5 & 4 \\
\cline { 2 - 6 } & 525 & 3.1 & 11 & 6 & - \\
\cline { 2 - 6 } & 870 & 2.4 & 8 & 7 & 4 \\
\hline \multirow{3}{*}{8} & 470 & 3.6 & 8 & 14 & 7 \\
\cline { 2 - 6 } & 525 & 4.9 & 19 & 13 & - \\
\cline { 2 - 6 } & 870 & 3.2 & 11 & 12 & 7 \\
\hline \multirow{3}{*}{10} & 470 & 5.8 & 13 & 23 & 10 \\
\cline { 2 - 6 } & 525 & 7.4 & 27 & 16 & - \\
\cline { 2 - 6 } & 870 & 4.1 & 15 & 15 & 9 \\
\hline
\end{tabular}


Table 2

\begin{tabular}{|l|l|l|l|}
\hline $\begin{array}{l}\text { Stimulation } \\
\text { Sources }\end{array}$ & $\begin{array}{l}\text { Wave- } \\
\text { length } \\
{[\mathrm{nm}]}\end{array}$ & $\begin{array}{l}\text { Band- } \\
\text { width } \\
\text { [nm] }\end{array}$ & $\begin{array}{l}\text { *)Power at } \\
\text { sample } \\
\text { position } \\
{\left[\mathrm{mW} / \mathrm{cm}^{2}\right]}\end{array}$ \\
\hline Blue & 470 & 17 & 100 \\
\hline Green & 525 & 30 & 50 \\
\hline IR & 870 & 45 & 180 \\
\hline
\end{tabular}

*) The power measurements refer to a DASH with 2 blue, 2 green and 3 IR LEDs

\begin{tabular}{|l|l|l|l|}
\hline & & $\begin{array}{l}\text { Wave- } \\
\text { length } \\
\text { range } \\
\text { [nm] }\end{array}$ & QE@ $\lambda$ [nm] \\
\hline Standard PMT & $\begin{array}{l}\text { ET EMD-9107 } \\
\text { PMT }\end{array}$ & $160-630$ & $\begin{array}{l}30 \% \\
@ 340\end{array}$ \\
\hline Red sensitive PMT & $\begin{array}{l}\text { Hamamatsu } \\
\text { H7421-50 }\end{array}$ & $380-890$ & $\begin{array}{l}12 \% \\
@ 800\end{array}$ \\
\hline & $\begin{array}{l}\text { Photometrics } \\
\text { Evolve 512 }\end{array}$ & $300-900$ & $\begin{array}{l}90 \% \\
@ 400-700\end{array}$ \\
\hline
\end{tabular}

\begin{tabular}{|l|l|l|}
\hline Standard Filters & Trade names & $\begin{array}{l}\text { Thickness } \\
{[\mathrm{mm}]}\end{array}$ \\
\hline UV & Hoya U-340 & 7.5 \\
\hline Blue & Schott BG-39+ Schott BG-3 & $2.0+3.0$ \\
\hline Neutral density & Schott NG9 -OD 7.5 & 5.0 \\
\hline
\end{tabular}


Figure 1

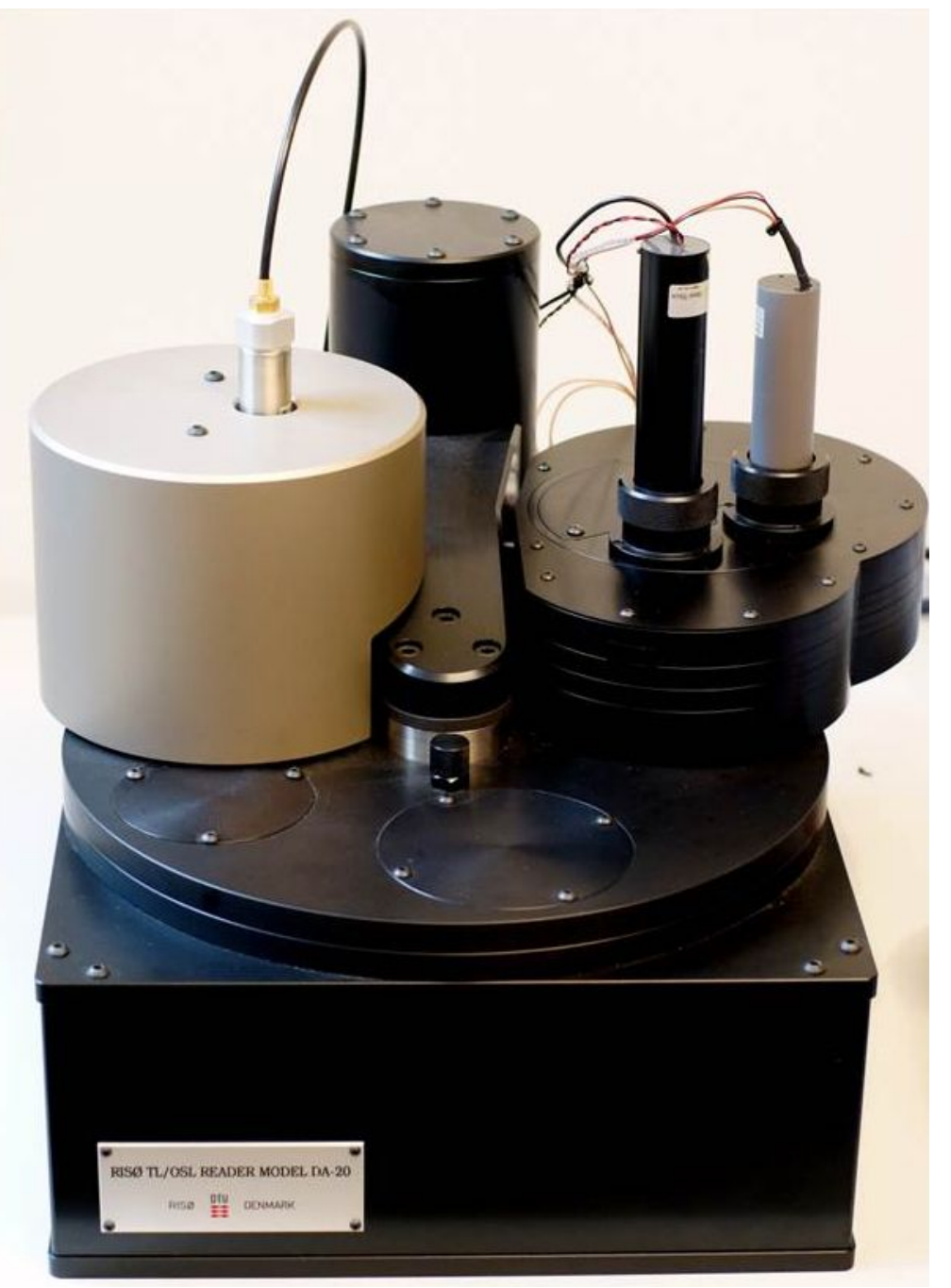


Figure 2

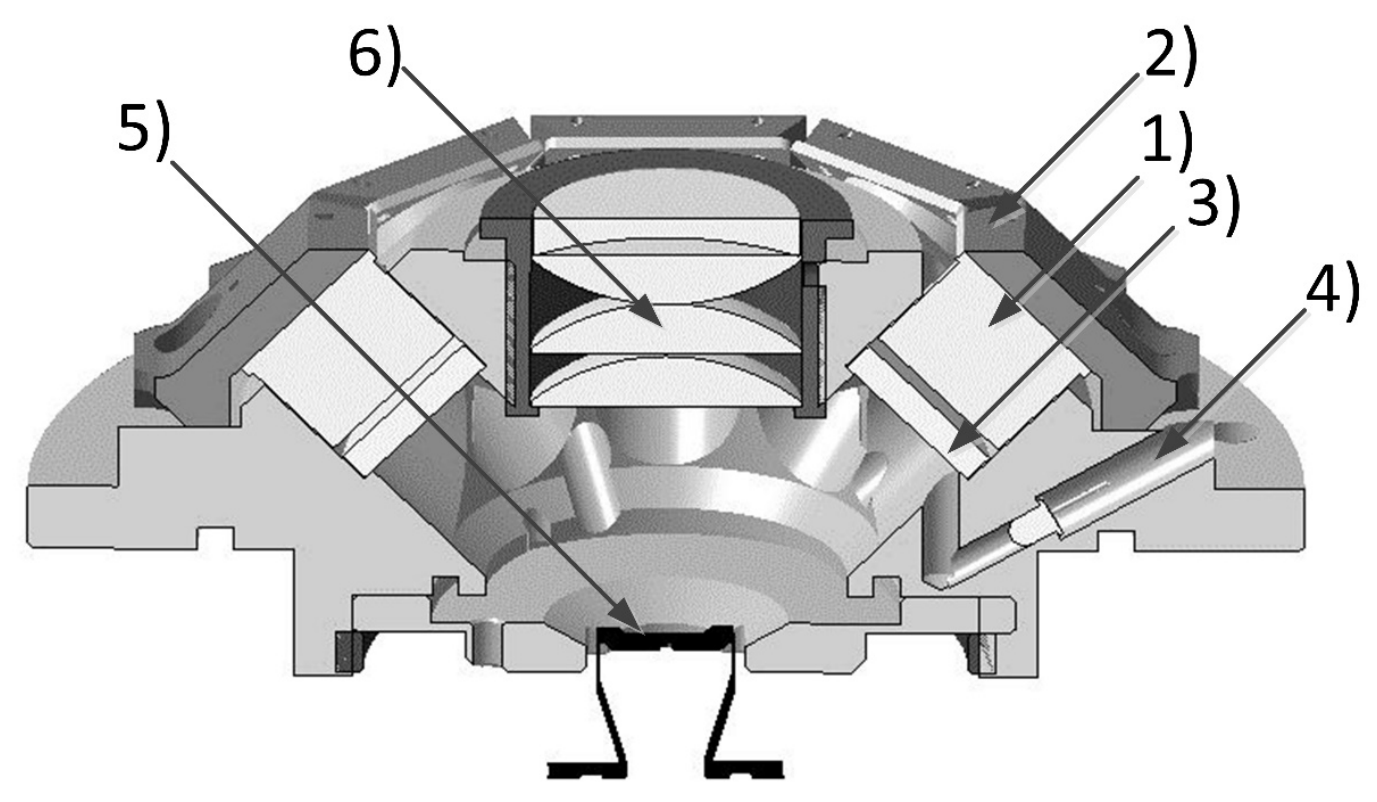









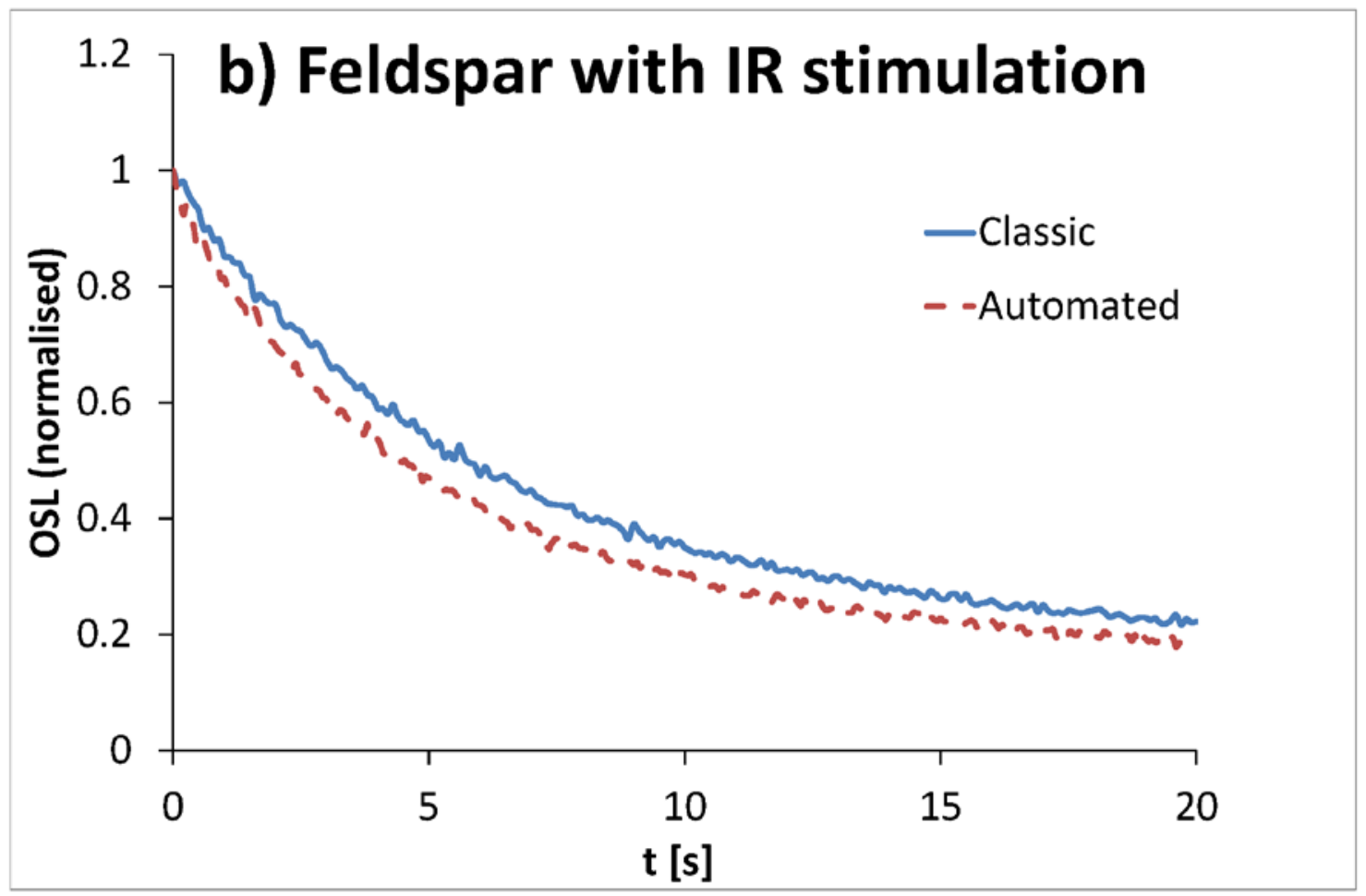


Figure 4a

Uniformity of DASH $470 \mathrm{~nm}$ LED stimulation field



100

110 
Figure $4 b$

Uniformity of DASH $525 \mathrm{~nm}$ LED stimulation field

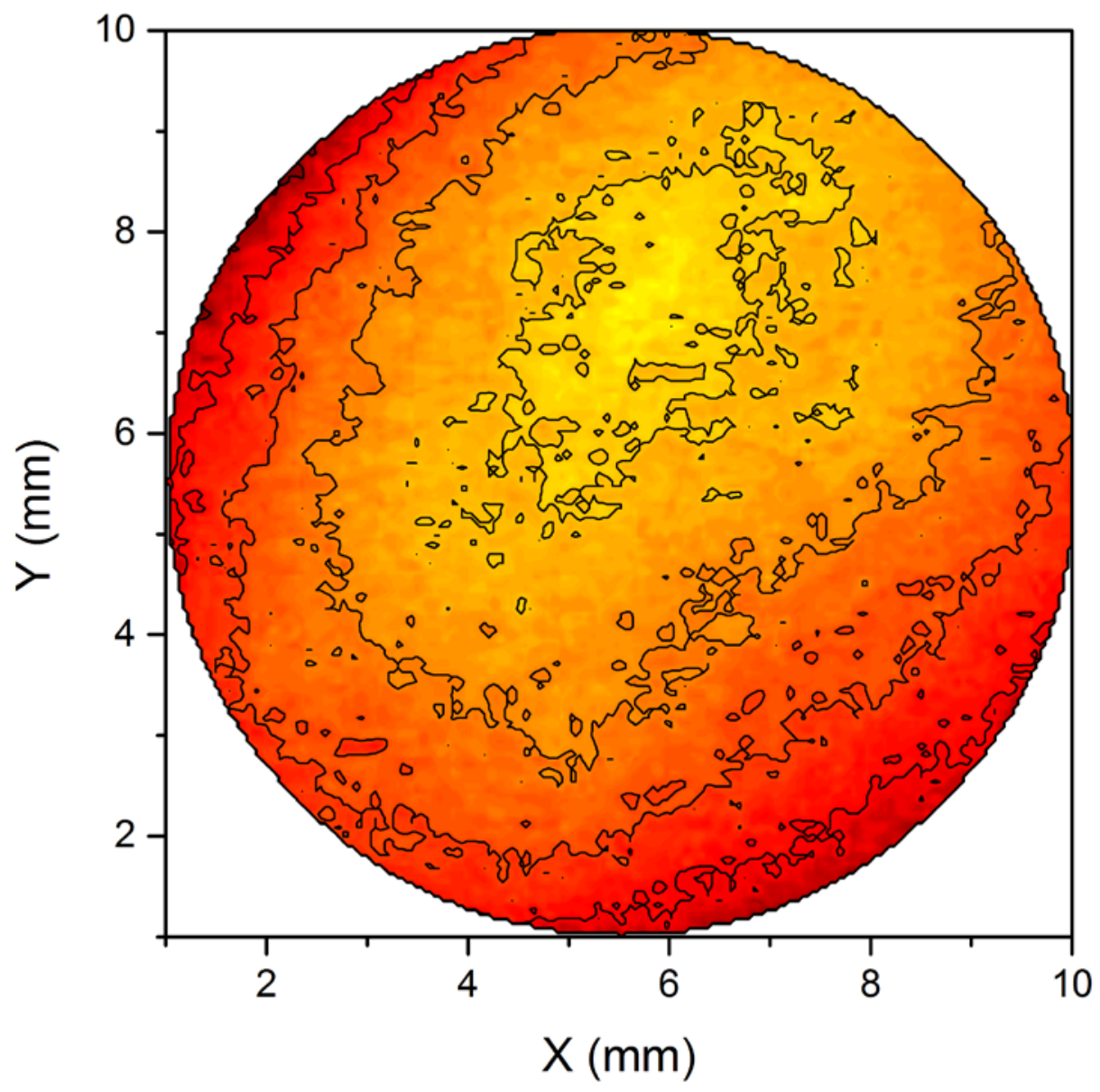


Figure 4c

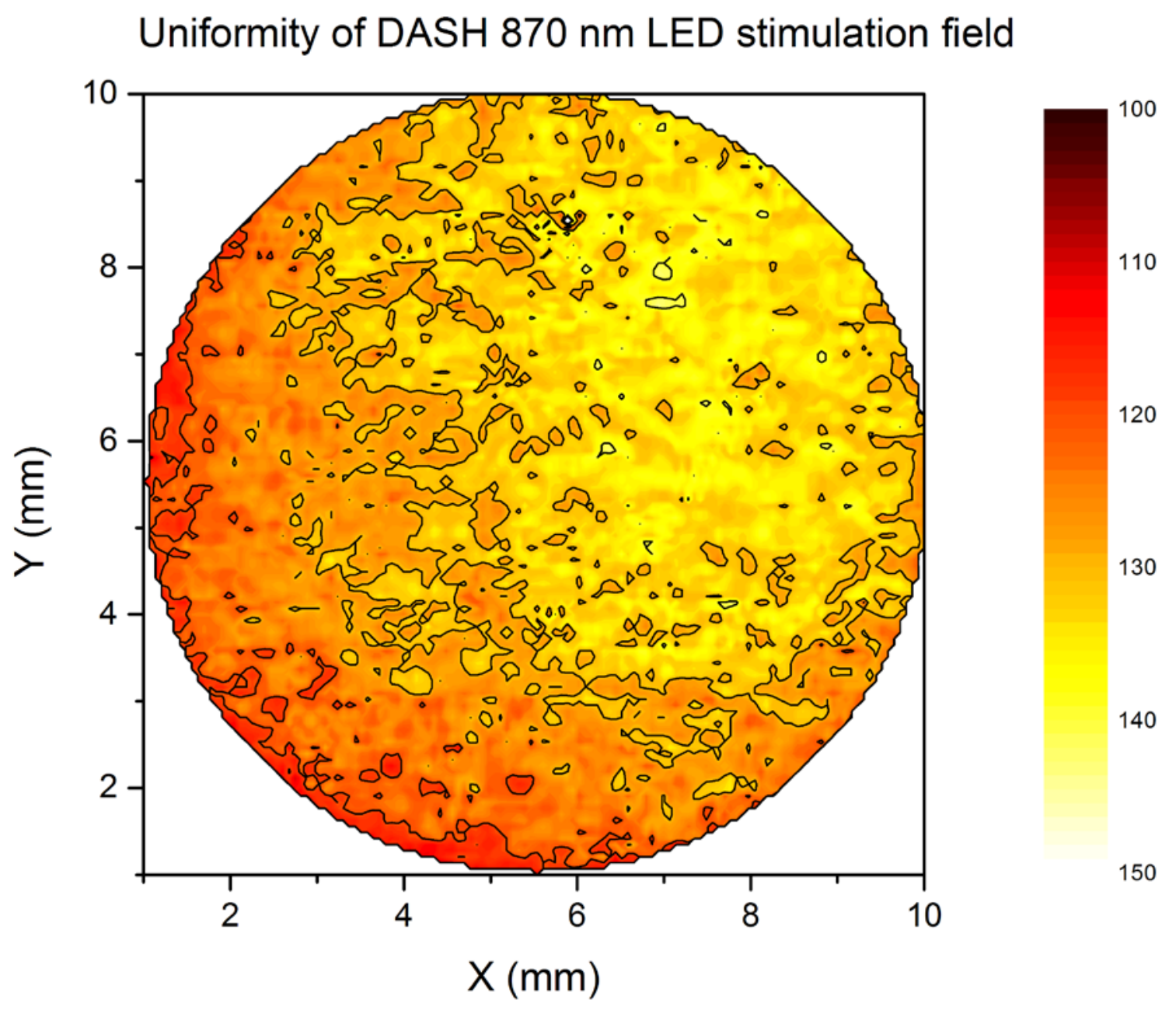




\section{Intensity profile along major and minor axis}

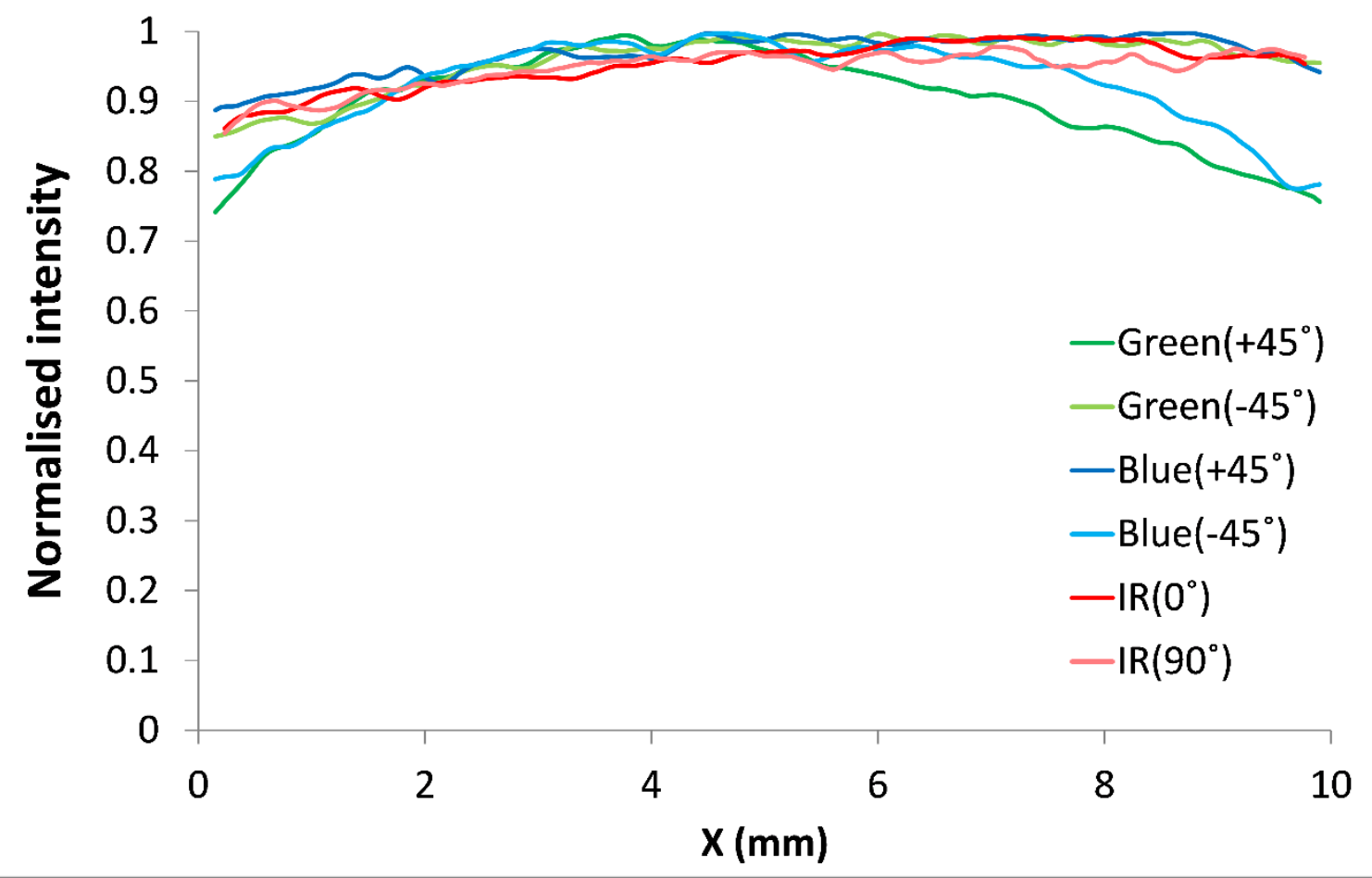


Figure 5
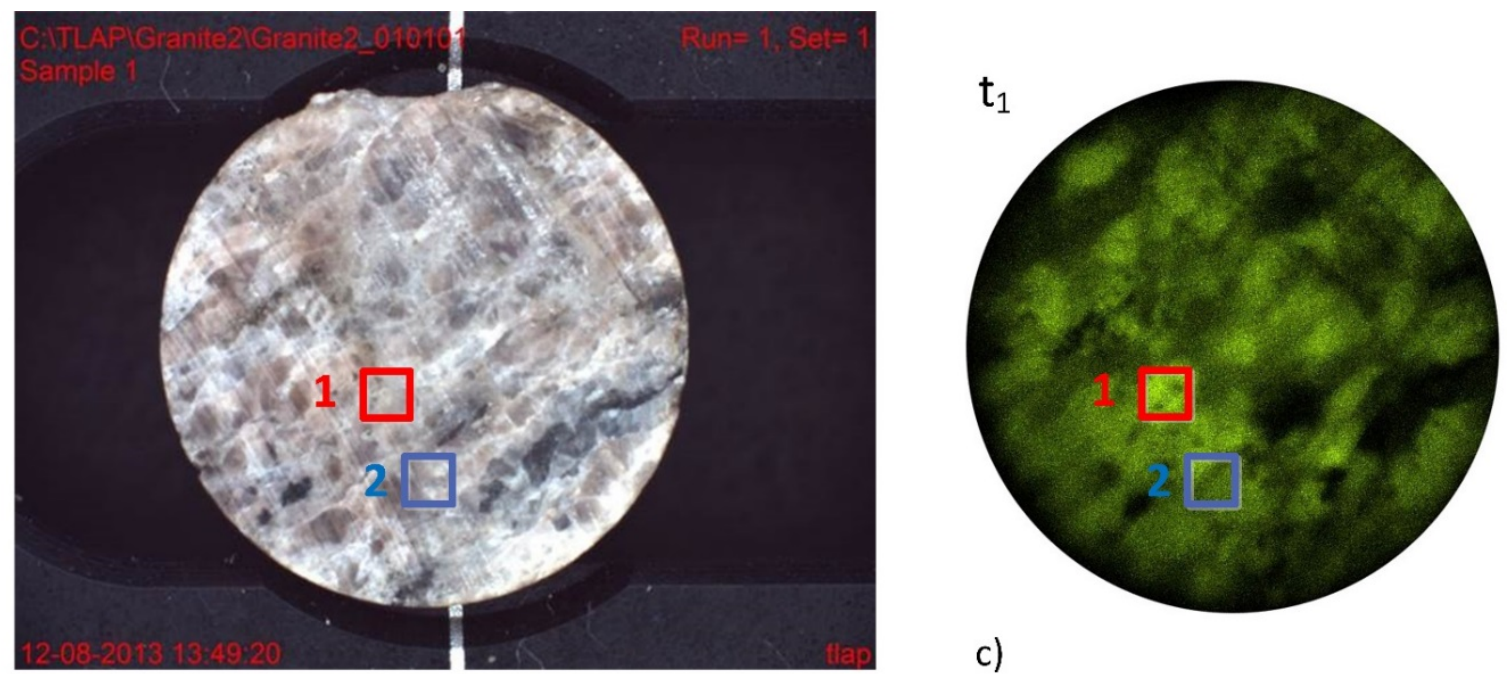

a)



b)

c)

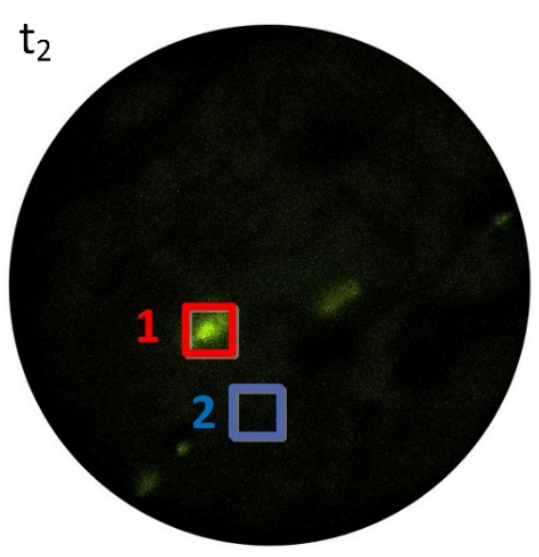

c) 